\title{
Impact of stabilizers on ice cream quality characteristics
}

\begin{abstract}
Ice cream is a frozen dairy dessert liked around the globe. Manufacturers use different types of stabilizers to improve the stability and mouth feel of the product. These stabilizers react differently with other ingredients and provide varying characteristics to the products. This article provides a brief overview of the mode of action of different stabilizers being used in the ice cream.
\end{abstract}

Keywords: ice cream, stabilizers, quality, mouth feel
Volume 3 Issue I - 2016

\section{Qamar Abbas Syed, Muhammad Shoaib Ullah Shah}

Department of Food Nutrition and Home Sciences, University of Agriculture Faisalabad, Pakistan

Correspondence: Qamar Abbas Syed, National Institute of Food Science and Technology, Faculty of Food Nutrition and Home Sciences, University of Agriculture Faisalabad, Pakistan, Email qamar.abbas@uaf.edu.pk; sqa1975@yahoo.com

Received: August 17, 2016 | Published:September 16, 2016

\section{Introduction}

Ice cream is a frozen product used as dessert and made from dairy ingredients emulsifiers, stabilizers and sweeteners i.e. sucrose or dextrose. Exceptions are with the usage of fruits or fruit pulp in the production of fruit based ice creams. ${ }^{1}$ In Pakistan there are many small and large ice cream manufacturing plants. Different estimates have shown that total installed capacity of different manufacturers including hotels, restaurants, recognized and unrecognized manufacturers in Punjab is 25 million liters per annum. In Baluchistan and Khyber Pakhtoonkhuwa provinces ice cream production has been estimated about 6million liters per annum. Till 1990, only 4 brand names were well known in the market which were IGLOO, POLKA, ROCO and YUMMY. Lever Brothers (Unilever) Pakistan entered in the market in 1995 which is a large multinational group with the brand name of WALL'S. In 2009, Engro foods limited have launched their ice cream under the brand name of "OMORE". It is a dairy ice cream manufactured in Sahiwal.

Unilever after launching their ice cream under the brand name of "Wall's" in 1995 have become the largest ice cream manufacturing plants of Pakistan after an amalgamation with Polka in May 1999. Wall's plant has the HACCP (Hazard Analysis Critical Control Points) and TQM (Total Quality Management) systems certification and it is situated at Bhai Pheru. Annual capacity of the plant is 30million liters. Pakistan has a market of 70 million liters of branded ice cream and this figure could be doubled if the non-branded section is added. $15 \%$ growth in the volume increase is observed by Unilever (Table 1).

The process of being whipped and being frozen are essential components for the developing acquired structure, creamy texture and getting palatability. Ice cream production is not recent trend but there are new developments since then. Since producers are emphasizing to shape a new frozen product, they are permanently stimulating food technologists to have new desired textures and to create a way that permit an idea that was not foreseen. ${ }^{2}$

The invention of new mechanical infra structure also gates innovations those were not implementable in past and that's why it makes new improvement based challenges. Since all the required characteristics in ice cream like low melting, nice flavor, palatable texture and homogenous entity etc. There are so much recipes and factors which affect the mouth feel and palatability of ice cream. Stabilizers transfer peculiar and essential attributes to the end product. In 1915, the word stabilizer was given to a group that was termed binders. They were also called improvers which reflected enzymes and mixture of enzymes and gummy materials ${ }^{3}$ (Table 2).

Table I Profile about demand of ice cream in Pakistan

\begin{tabular}{ll}
\hline Year & Total demand (in million liters) \\
\hline 2010 & 172 \\
2011 & 219 \\
2012 & 267 \\
2013 & 271 \\
2014 & 275 \\
2015 & 280 \\
\hline
\end{tabular}

Table 2 Formulation of typical ice cream

\begin{tabular}{ll}
\hline Ingredient & Percentage \\
\hline Fat & 12 \\
Solids not Fat (SNF) & 12 \\
Sweetener (Sucrose) & 10 \\
Flavor & A few drops
\end{tabular}

\section{Hydrocolloids}

Those bodies that show these substances are mega molecules majorly polysaccharides, which are asked to interact with water. Relation with water permits some compounds to react with proteins and lipids in ice cream. Stabilizers consist of 103 monomer units and molecular weights that of $₫ 105-106$. The basic cause for utilizing stabilizers in dairy is to have homogeneity in body and texture; kills ice and growth of that of milk sugar when stored. Especially during time when temperature is not constant; gives uniformness, 
and acceptable extent of shape persistence when melting. They also contribute to viscosity, strengthen the protein in the ice cream to escape whey separation, assist in suspension of product.

Stabilizers should be clean, pleasant flavor, should not stick to other particles, promote good melting of the product and impart good texture when consumed. In spite of being natural under European legislation they are assumed food additives, that's why having associated E numbers. The best stabilizer must be non hazardous, should be already dispersed in the ice cream, not emit heavy viscosity and melting, not cause stains, contribute ice cream with required characteristics, be cheap and reasonable and not give off flavor to the product. The quantity and type of stabilizer used in ice cream dependent on its profile.

Ratio of mix, and milk to be used; time to process, and reasonable air pressures; storage conditions also determine efficiency of stabilizers. Commonly $0.1-0.5 \%$ stabilizer is used in the product of ice cream. Ice creams with high fat and solids-not-fat (SNF) contents as well as ultra high temperature (UHT) treated products need much fewer stabilizer than others. The reason is that high contents of fat and SNF increase the product viscosity and improve stability of the product. Similarly UHT treatment also improve product stability and hence lessen the stabilizer needs.

Hartel ${ }^{4}$ rediscovered crystals of ice during production of product and reported that the effects of different factors in this phenomenon were noticeable. These factors include time, temperature, conductance properties of the ice cream and the container as well as distance for heat to travel.

\section{Methodology of crystallization}

Serum and stabilizers reactions and serum stabilized inefficiency have been studied by Sybre et al. ${ }^{5}$ They unveiled the process of stabilization of serum in product and relevant items with a look on colloidal science. Dickinson ${ }^{6}$ disclosed hydrocolloids at intervals and the work of such hydrocolloids on attributes of dispersions, emulsifier capability of stabilizers and serum- stabilizers junctions.

\section{Role performed by stabilizers}

i. To stabilize the emulsion

ii. To aid in suspension of liquid flavors

iii. To stabilize the air bubbles

iv. To prevent lactose crystal growth

v. To suppress the effects of altitude abuse

vi. Ultimately reduces the occurrence of shrinkage

\section{Types and characteristics of stabilizers}

These are as follows: Gelatin which is animal protein, is first substance used as an ice cream hydrocolloid, However this mostly alternated by polysaccharide hydrocolloids now a days in ice cream production. Important stabilizers and their properties are given here.

Gelatin (E 441): This is a bit expensive stabilizer and productive at rate of $0.3-0.5 \%$. But it does not stop the adverse effects of heat shock. It is not recommendable to some ethical regions and vegetarian surroundings. Gelatin is basically adopted as a gelling agent making transparent gels on cooling if temperature is below about $35^{\circ} \mathrm{C}$, which deforms at low temperature to cause melting in the product with good flavor scores. Furthermore, due to the amphiphilic in nature it is overall major additive utilized for gelling, current scenarios about usage of this an animal based product which yield Creutzfeldt-Jakob disease. $^{7}$

Likewise, the need created by vegetarians and some ethical groups wish has been aroused by them to stimulate the serious quest search for other options. Gelatin is not homogenous but it consists of glycine, proline and 4-hydroxyproline residues. It is utilized in low fat-products foods to give simulate the feel of fat and to give volume. Gelatin depicts thixotropic behavior.

Guar gum (E 412): It is obtained from the seeds of Cyamoposis tetragonolba. It is being grown in Pakistan for centuries and in the USA. It is less costly and comprehensively retards unpleasant effects in ice cream. It readily becomes dispersed and does not yield heavier viscosity in the product. Commonly $0.1-0.2 \%$ is required in a premix and, that's why this is imagined to be a powerful hydrocolloid. It has higher molecular weight $(50,000-8,000,000)$.

Hereby galactopyranose and mannopyranose units combined through glycoside linkages known as galactomannan. The mannose: galactose ratio is about 2:1. It is richly efficient water-thickening body in industry. It is a GRAS stabilizer (184.1339 in Title 21 set by the U.S. Code of Federal Regulations).

It possess higher ratio of dietary fiber ( $78 \%$ to $87 \%)$. It has fewer prices for different food applications. It is readily soluble in water and yields hazy, reasonable $\mathrm{pH}$. Solutions are heat stable from $\mathrm{pH} 4$ to 11; viscosity is high from pH 5.9 to 7.8. Guar gum is in accordance with nonionic and anionic gums, giving beneficial synergistic activity.

They depict increased alignment of constituent molecules of system. The extent of alignment becomes higher as we increase its amount. Presumably they do not show yield stress characters. Now days, guar gum is mostly used in ice cream as stabilizer. Guar is given upper hand due to its low price and the texture it gives to the ice cream. It gives hydration very well in cold solution and, therefore centralized 2 mannose and 1 glucuronic acid on every other glucose at the $\mathrm{C}-3$ point. Almost half the terminal mannose units proceed with a pyruvic acid on an acetyl group at C- $6{ }^{8}$

Sodium carboxymethyl cellulose (E 466): This chemically changed gum is a linear, has big chain, soluble in water, and anionic based hydrocolloid. Pure CMC is a whitish creamy with no taste, no odor and free-flowing. CMC creates weaker gels by themselves but gels good with k-carrageen and LBG. It is a stronger hydrocolloid and only $0.1-0.2 \%$ is required in ice cream. It gives body which looks good and imparts chewiness to product.

Locust Bean Gum (carob bean gum) (LBG) (E410): It is extracted from Ceratonia clique, which is grown in the Mediterranean area. This strongest hydrocolloid is utilized at $0.1 .5-0.3 \%$ and avoids phase separation.

LBG is only being soluble in cold solution and it should be heated above $85^{\circ} \mathrm{C}$ to make it hydrated completely. It yields uniformity, better, and desired viscosity that is not disturbed by agitation.

i. This cools with uniformity and permits good whipping 
ii. It gives good quality heat-shock resistance.

iii. It never emits taste and flavor related attributed.

iv. It gives cryo-gel that is productive against cryo-protection

After combining with xanthan, it gives gels. In the presence of kepa-carrageenan, LBG forms gel. The synergistic mechanism with xanthan yields important benefits such as reinforcing carrageenan gels, flexible texture and avoidance from whey separation. It increases pleasant texture along with a better flavor scores. When utilized alone, this cause syneresis not like that of guar, it should be heated to $80^{\circ} \mathrm{C}$ for fully hydrated texture which is attained during pasteurization.

Solutions of LBG are non- uniform and have less yield digits. It flows when slightly shear is exerted. In LBG, the ratio of mannose to galactose is bigger than in guar gum.

It is reported for its thickening characters since old times. The Egyptians used its paste to bind bandages onto dead bodies. But it happened just in $20^{\text {th }}$ century which made industrial entity. It is basically the underground endosperm of Ceratonia siliqua. The ratio of mannose: galactose is about 4.1:1.6. This is GRAS substance under section 588.7347 in Title 22 by the U.S CFR.

Carrageenan (Irish moss) (E 407): It was initially derivative of Chondus crispus. Its chief sources include, Eucheuma cottonii and E. spinosum, they are industrially shaped in the Indonesia, Tanzania and Philippines. The pull out of Kappaphycus alarezii is exactly pure kappa carrageen, while the take out of E. denticulatum is a somewhat wholesome iota carrageenan. The grains of Gigartinacean algae, Gigartina skottsbergii, Sarcothalia crispate, and Mozzarella laminarioides are gelling carrageenans that are weakest and less proactive with kappa casein in milk than $C$. crispus extracts. ${ }^{9}$ These gelling carrageenans are set up by polymers of kappa carrageen.

Xanthan (E 415): This polysaccharide is attained by the expansion of Xanthomonas campestris in culture. Its merge amid guar gum and locust bean gum makes a useful stabilizer for dairy products. An amalgamation of xanthan gum with sodium alginate is observed to dole out as a milk wobble stabilizer. It is permitted for GRAS food stuff under section 172.675 in Title 22 set by the U.S CFR172.675.

It has elevated stabilizing attributes exceptional freeze/thaw and well-suited with roughly all commercial thickeners. It can be detached by unification with skim milk. It is constantly worn in recipe with other gums.

Local Hydrocolloids: They are used as ice cream stabilizers. Salep, is attained by grinding dried tubers of orchids and is functional as an indispensable ingredient for the construction of established ice cream in Iran and Turkey.

This category of ice cream is called kahramanmaras Turkey, different from familiar ice cream in its high sugar level, mouth feel, and muggy gooey body, above all due to salep being added. Maras's type ice cream is presented solid and a dagger ought to be worn during utilization, because its exclusive textural attributes. In comparison to other stabilizers, salep is utilized in highest quantity, normally 0.76 $2 \%$, in ice cream recepie. Totaling to stabilizing attributes salep has wellbeing advantages. Salep consist roughly 13-49\% higher polysaccharides. ${ }^{10}$
Glucomannan: It is off the record as a hydrocolloid. It takes in $150 \mathrm{~mL}$ of water per $10 \mathrm{gram}$. In accordance to Farshoosh et al. ${ }^{11}$ salep cultivars developed in Iran come in two shapes, one with cleft and the other with branched tubers. Even though foodstuffs arranged using only salep emitted superior contrast comparison to ice cream having $\mathrm{CMC}$, all differences are not understood.

\section{Alginates}

Alginate: Alginate is a naturally occurring polysaccharide obtained from different species of algae and consisted of beta-D-mannuronic and alpha-L-guluronic acids. The composition of the alginate may vary with the source of the alginate. There are two types of alginate calcium alginate and sodium alginate. This also affects its properties and its use as encapsulated material. It is being used for the encapsulation on a large scale. ${ }^{12}$ Due to simplicity, non-toxicity and economical price of the calcium alginate and sodium alginate is being preferred over the others. ${ }^{8}$

Brown algae are the source of the alginate. It occurs in the cell wall of the brown algae. Its function is to provide the strength and flexibility to the plant cell. Alganic acid is added to make stable water alginate different salts are also added this gives the sodium or calcium alginate. It is reported that for the encapsulation of microbial cells alginates have been extensively used..$^{13} \operatorname{Dogan}^{14}$ found that alginate starch enhances the survival of the probiotic in simulated gastrointestinal conditions also in yoghurt.

On the other hand, alginate has some disadvantages. For example, alginate beads are sensitive to the acidic environment which is not appropriate for stomach conditions. Another disadvantage is that it's scaling up is a difficult process and the beads are very porous that are not suitable for the protection of the bacteria. However, the defects can be compensated by mixing alginates with other polymer compounds, covering the capsules by another compound or applying different additives. ${ }^{15}$

Starch is being commonly used and it has shown that this method results in an enhancement of probiotic encapsulation effectiveness. ${ }^{1}$ Sodium alginate has been used for the encapsulation of the bacterial cell. Lots of encapsulating materials are available but sodium alginate is more suitable for the encapsulation of probiotics. It is considered as non-toxic. It is also used as additive in the food industry. It is also popular in the pharmaceutical industry. It is economical, simple to use, stable to heat, forms jell readily, high retention of viability and it is naturally occurring. ${ }^{16}$

Calcium alginate is very useful for giving the protection to bacteria and the bactericidal effects. It also improves the sensory properties of the mayonnaise. ${ }^{17,18}$ Alginates are utilized in a extensive assortment of implications like stabilizers. The initial methodical study on the withdrawal of alginates from red seaweed ended by the chemist E.C. Stanford in the $19^{\text {th }}$ century. Industrial cultivars of alginate are obtained from red algae as well as the colossal kelp Macrocytis. These are not homopolysaccharides finished up of block of $\beta-(1 \rightarrow 5)$-linked $\mathrm{c}$-mannuronic acid and $\beta$-(2 $\rightarrow 4)$-linked m-guluronic acid.

Alginate building unit: Alginates are utilized for a more than 53 years. From side to side selection of rating and recipe, the stream distinctiveness of alginates can be monitored. The watery solutions of alginates have shear-thickening properties called increased alignment. 


\section{Impact of stabilizers on flow characteristics}

Rheology is a stem of science troubled with the opus and configuration of smooth and deformed foods. Information of the distinctiveness of products are imperative for superiority control, body and the collection of the appropriate instruments. Horizontal body and cool feel, these are the nearly all frequently much loved characters of ice cream through utilization, could be gained by an ice cream with finest flow characters. Ice cream reveal non-uniform increased alignment denotation that there is a no linear affiliation stuck between shear constant worry and shear rate, with the noticeable glueyness declining with escalating shear rate. The increased alignments have been associated to the shear rate. Commonly, the power law is worn to in shape the flow characters of the ice cream.

$$
\tau=K \boldsymbol{\gamma}^{\bullet} n
$$

The standards $n$ and $K$ are vital flow characters of liquid foods, as the rhelogy of foods is distinguished in stipulations of these parameters. The slighter the $n$ value, the superior the going away from uniform actions and, that's why, the superior the alignment. The consistency values are painstaking to be assessing of the gelatinous environment of the food, more with stabilizer quantity. This is observed that gums showed larger enhance in non-Newtonian deeds than anionic ones. Viscosity is the majority imperative flow characteristic of ice cream and frozen bit of ice cream, is prejudiced by recipe. ${ }^{17}$

The viscosity of ice cream place from first to last sequence, predominantly stabilizer substance. Even if this is commonly tacit that viscosity is vital to communicate enviable traits of ice cream, the explicit flow characters compulsory not absorbed properly. Commonly since the viscosity is more the battle to melt and the velvetiness of texture is also more, except the rate of air incorporation become less. Frequent scientists have unveiled the flow characters of ice cream. Goff et al. ${ }^{19}$ observed $(n)$ of ice cream 0.7, even though other observed it from 0.39 to 0.88 . Earlier it was shown that more amount and less temperature gives more alignment. Kaya and Tekin observed that salep amount has a more impact on viscosity.

\section{Impact of stabilizers on phase separation}

As nearly all stabilizers of industrial cause are unfit with serum, phase separation happens. Causing modify of purposeful deeds of the proteins, an illustration partition of a clear serum, and a thrashing of enjoyable superiority in the ice cream. This happens in those who are ready to serve.

Diverse gums have unusual special effects on phase separation, Thaiudom ${ }^{20}$ observed that between the stabilizers observed, guar gum is the the largest part ill-assorted with serum then LBG. Schorsch et al. ${ }^{21}$ observed that adding up sucrose caused good impact effect on serum and strength of the locust bean gum phase. The consequence of molecular configuration on phase severance is noticed by Bourriot et al. ${ }^{22}$

\section{Impact of stabilizers on volume increase}

Ice cream is aerated and defined as frozen texture. More ice cream quantity is one responsibility of stabilizer, bring about from beginning to end more viscosity and persisting the air molecules. The quantity of air in product is central since it affects superiority and income saves for also since of authorized principles that should be gained.

Moreover, the air cubicle organization has confirmed to be the chief agent affecting melting, and the flow characters in the frozen condition, this is linked to creaminess. Slighter air cell perk up the superiority concerning these three factors. Harte ${ }^{23}$ observed impacts of unit operations on expansion of air cells. Alteration in stabilizer that consisted of $13 \%$ carrageen, $37 \%$ guar gum, and $51 \%$ carboxy methyl cellulose (CMC) did not affect freeze thawing. Adding up stabilizer, but less, air small room volume compare to an analogous product ended devoid of stabilizer. Modifications in air chamber volume can be frankly contributed to modifications in flow characteristics of the product.

Shrinkage and Expansion

i. Different protein source

ii. Low-fat ice creams

iii. Worldwide geographically distribution of product

iv. Altitude downfall

v. Smallest air cells

vi. Smaller crystals of icy structure

vii. Un appropriate mixing

viii. Heavy fat being agglomerated

ix. Weaker body

\section{Impact of stabilizers on thermodynamic characteristics}

LSC observed that thermodynamic characteristics like glass transition and ice amount resolute by the melting endothermic are alike in system with and devoid of the being there of a stabilizer. But such parameters gave battle to thermal de stability and drastically exaggerated the thermal mechanism.

This is depicted that ice cream with higher locust bean gum has the higher conduction. Product with high LBG freeze quickly, since the moderately lower contents of water activity makes it lesser viscous.

The lesser heat of fusion is as there is more the water binding ability of hydrocolloids.

\section{Cryoprotectivity of stabilizers}

The cryoprotectivity can be underlined by following.

Viscosity and molecular mobility: It states that more viscosity since more adding up due stabilizers are associated to the ice crystals of ice and their growth. But in spite of more work, there was never any evidence with regard to correlation among viscosity and crystallization. Budiaman ${ }^{24}$ observed the linear rate of re crystallization in different stabilizers solutions at -4 to $-7^{\circ} \mathrm{C}$.

According to Harper et al. ${ }^{23}$ viscosity is not correlated well with stabilizer mechanism and guar gum is not booster of crystallization under in vitro situations. Bollinger observed direct relation among universalized "breakpoint" obvious viscosity followed by crystallization. They planned that a few ways of stabilizer authioncity with regard to crystallization fortification comes from the more alignment which is observed from frozen contents of stabilizers in the unfrozen step phase of product as to hypo-entanglements.

This is being recommended that while we study the correlation among water being mobile and crystallization is cooperative in 
thought fulling the system of stabilizer achievement and also scheming crystallization.

\section{Impact of Stabilizers on Melting}

While ice cream is in the shape of a funnel, melting is of supreme value to the customer. Slower melting, slower draining of serum, nicer shape persistence, and lesser foam disruption are preferred imperative eminence factors of product.

\section{Quality parameters}

i. Controlled melting of finished product

ii. Controlled draining of serum

iii. Good Shape retention

iv. Controlled foam collapse

v. Messy situation results if melting is over

\section{Impact of stabilizers on sensory characteristics}

Stabilizers affect the sensory profile of product. Even though here are numerous gossip commerce with the consequence of stabilizers on texture insight and mouth feel of ice cream. There are deficient untried figures on the meticulous exploit of stabilizers on precise sensory mechanism of product.

A case study was performed showing following results

Ice creams having $0.25 \%$ xanthan gum

i. Better pseudoplasticity

ii. Better flavor release

iii. Product with $0.3 \%$ sodium alginate

iv. Higher viscosity

v. Better perceived pseudoplasticity

vi. Better texture

vii. Low melting

viii. Extended shelf life

ix. Improved quality

$\mathrm{x}$. Consumer preference

\section{Why hydrocolloids as stabilizers}

i. Colloidal system having the colloidalized particles being hydrophilic polymers discrete in water

ii. Average consumer first choice.

iii. It is natural source to be in use as stabilizer

iv. $\square 10^{3}$ monomer units

v. $\square 10^{5}-10^{6}$ Molecular weights

Natural stabilizers are from natural extractions and are refined by natural ways like mechanically processed and extracted by warmth processes. These natural stabilizers take in guar gum, locust bean gum, xanthan gum, carrageenan, starch, sodium alginate etc. Gelatin, a protein which is animal based is utilized more or less utterly in the ice cream being a stabilizer, however has progressively been alternated with stabilizer of plant based cause their more productivity and less, reasonable $\mathrm{pH} .{ }^{25}$

\section{Fermented glutinous rice as stabilizer}

Fermented glutinous rice is primed by with the roasting methodology. In grounding of tapai pulut, $500 \mathrm{~g}$ of glutinous rice is wash out and saturated for $2 \mathrm{~h}$ before being cooked. The ripe glutinous rice is then roasted for $25 \mathrm{mins}$. Now, the glutinous rice is partially being cook. Just about $250 \mathrm{ml}$ of water is further put into the roasted glutinous rice and varied well sooner than it was roasted yet again for 10 mins. The roasted glutinous rice was chilled at $37^{\circ} \mathrm{C}$ prior to $0.2 \%$ of 'ragi' was multiply in excess of the glutinous rice and assorted well. Fermented glutinous rice was attained after the being fermented for 52 to $74 h$.

\section{Preparation of fermented glutinous rice}

Giessen $^{26}$ prepared ice cream by mixing $140 \mathrm{~g}$ yolk of eggs and $160 \mathrm{~g}$ castorized sucrose pending substantial and beam. About $550 \mathrm{ml}$ of spanking new milk was steadily overcooked and mixed with the mixturized eggs. This was heated for $25-35$ seconds at $80^{\circ} \mathrm{C}$ for pasteurization before each stabilizer was included.

Dissimilar compositions of tapai pulut ice creams shaped such as Formulation 1(b1) (15\% tapai pulut), Formulation 2 (b2) (20\% tapai pulut), Formulation 3(b3) (30\% tapai pulut) and Formulation 4(b4) ( $40 \%$ tapai pulut) were urbanized and sensory assessment of the overall reception was performed. ${ }^{27}$

\section{Fourier Transform Infrared (FTIR) analysis}

It was utilized to mark importance of the functional derivatives gained from the fermented glutinous rice ice cream. In it products were located on the taster booth of the spectroscope. The FTIR spectra was gained utilizing silver-gate ATR in FpS 9000 FTIR spectrophotometer with a DpGS detector (Nicolet 390). The spectra was observed at the mode of absorbance from 2000 to $3000 \mathrm{~cm}^{-1}$ having a resolution of $5 \mathrm{~cm}^{-2}$ with 130 added up scans. ${ }^{28}$

The firmness of product is relevant to the arrangement, so this will impact receipt level of the product. consequences gained depicted that the steadfastness of b3, b1, b2 and b4 products appreciably amplified $(p<0.05)$ by the adding up conventional stabilizers The enlarged of steadfastness values of the product was because there was the starch comprised in the rice pellet that got thicken product. ${ }^{29}$

The maximum gain for steadfastness was attained by the adding up guar gum $(5408.70 \mathrm{~g})$ then CMC (4938g) and finally xanthan gum $(2480.73 \mathrm{~g})$ to the fermented glutinous rice ice cream. Ice cream with more guar gum was more steady than the without it whereas b3 and xanthan gum was not appreciably diverse from any other. The superior steadfastness score for the ice cream with CMC added was since in cooperation with stabilizers it was found stable. ${ }^{30}$

\section{Overrun properties}

It was observed that the adding up xanthan gum into fermented glutinous rice ice cream offered the uppermost value $(64.86 \%)$ then by guar gum $(58.06 \%)$ and then CMC (43.87\%) in overrun. Similarly, the accumulation of these stabilizers into fermented glutinous rice ice 
cream drastically augmented $(\mathrm{p}<0.05)$ the overrun values. There was a noteworthy difference $(\mathrm{p}<0.05)$ in overrun value sandwiched between control and S1, S2 and S3. The overrun assessment was affected by the incorporation of air to the ice cream freezer. The air froth in these products was straight away attentive in milk protein by recrystallized fat being coalescence. ${ }^{31}$

\section{Modified starches as stabilizers}

The sway of industrial stabilizers (Fulfil 400, locust bean gum; LBG and guar gum) and modified starches (octenyl succinic anhydride starch; OSA, acetylated starch; AS and hydroxypropylated starch; HPS) on stability of foam and the increase in volume of product was evaluated. The obvious viscosity of a stabilizer at various levels was investigated to evaluate their aptitude for decreasing collapsing of foam, which resulted in high stabilized foam. 24-hours after being aged product which contained $16 \%$ butter oil and dissimilar stabilizers was observed to guesstimate the upshot of hydrated stabilizers on the product viscosity. The increased volume of product after being whipped was evaluated. The consequences depicted that the viscosities increased in the subsequent order: LBG $>$ OSA guar gum $>$ fulfil $400>$, guar gum $>$ HPS. Generally, it was assumed that LBG and xanthan gave more viscosity and stabilized foam to product and modified tapioca starches such as OSA and HPS exhibited no noteworthy disparity $(\mathrm{p}>0.05)$ in the volume.

\section{Why Modified Starches}

i. They were lower in cost

ii. Better texture

iii. Lower melting

iv. Less freeze thawing

v. Good mouth feel

vi. Source of protein

vii. Gateway to unconventional stabilizers

viii. Esterification and etherification based stabilizers

ix. Improved foam stability

\section{Drawbacks caused by stabilizers}

Even though stabilizers have useful role in dairy emulsions, their too much use might generate hurdles. These restrictions comprise

i. Phase separation

ii. Change in functional behavior of the proteins

iii. Heavier and soggy body

iv. Heavier melting

v. Heavier texture

vi. Prone to oxidation

\section{Therefore it is recommended that}

i. Synergistic mixtures are required ii. Trial and error method is required

iii. To achieve good quality product with good blending of appropriate stabilizer with regard to respective market niche.

\section{Acknowledgements}

None.

\section{Conflict of interest}

The author declares no conflict of interest.

\section{References}

1. Alvarez VB, Costello M. Ice cream and related products. The Sensory Evaluation of Dairy Products. 2nd ed. Business Media, LLC, NY, USA: Springer Sci; 2009. p. 1-163.

2. Bahram P, Thomsan CSA. Optimization of functional properties of three stabilizers and $\kappa$-carrageenan in ice cream and study of their synergism. $J$ Agri Sci Technol. 2013;15(4):757-769.

3. Cruz AG, Antunes AG, Sousa AL. Ice cream as a probiotic food carrier. Food Res Int. 2010;42(9):1233-1239.

4. Hartel RW. Crystallisation in foods. Gaithersburg, MD, Nertherlands: Aspen Publishers; 2001

5. Syrbe A, Bauer WJ, Klostermeyer H. Polymer science concepts in dairy systems-an overview of milk protein and food hydrocolloid interaction. International Dairy Journal. 1998;8(3):179-193.

6. Dickinson F, Robert HP. The effects of the combined use of stabilizers containing locust bean gum and the storage time on kahramanmaras-type ice creams. Int J Dairy Technol. 2013;56(4):223-228.

7. Jagdish KS, Arvind SA, Ashok RB. Utilization of guar gum as stabilizer in ice cream. Int J Curr Microbiol App Sci. 2015;4(1):284-287.

8. Khodaparast MH, Razavi SMA. The effect of carboxymethyl gums on the physiochemical and sensory properties of typical soft ice cream. J Int Dairy Technol. 2009;62(4):571-576.

9. Schmitz B, Thomsan CSA, Amante RD. Cassava starch functional properties by etherification-hydroxypropylation. Int J Food Sci Technol. 2009;41(6):681-687.

10. Sofjan RP, Hartel RW. Effect of overrun on structural and physical characteristics of ice cream. J Int Dairy. 2011;14(3):255-262.

11. Farhoosh R, Riazi AA. Compositional study on two types of salep in Iran and their rheological properties as a function of concentration and temperature. Food Hydrocoll. 2007;21(4):455-460.

12. Rowley JA, Madlambayan G, Mooney DJ. Alginate hydrogels as synthetic extracellular matrix materials. Biomaterials. 1999;20(1):45-53.

13. Hansen LT, Allan-Wojtas P, Jin YL, et al. Survival of Ca-alginate microencapsulated Bifidobacterium spp. in milk and simulated gastrointestinal conditions. Food Microbiol. 2002;19(1):35-45.

14. Donati S, Holtan YA, Borgogna M. New hypothesis on the role of hydrocolloids on ice crystals in ice cream. Int $J$ Biol Macromol. 2008;6:1031-1040.

15. Krasaekoopt W, Bhandari B, Deeth H. Evaluation of encapsulation techniques of probiotics for yoghurt. Int Dairy J. 2003;13(1):3-13.

16. Lee KY, Heo TR. Survival of Bifidobacterium longum immobilized in 
calcium alginate beads in simulated gastric juices and bile salt solution Appl Environ Microbiol. 2000;66(2):869-873.

17. Soukoulis C, Lebesi D, Tzia DC. Enrichment of ice cream with hydrocolloids: effects on rheological properties. $J$ Food Chem. 2009;115(2):665-671.

18. Klessmen KS, Mudahar JS, Singh GS. Flow behavior characteristics of ice cream mix made with buffalo milk and various stabilizers. Plant Foods for Human Nut. 2012;57(1):25-40.

19. Goff HD, Davidson RS, Cottrell JL, et al. The effect of stabilizers on the viscosity of an ice cream mix. J Sci Food Agri. 2008;31(10):1066-1070.

20. Thaidoum AC, Razavi BM. Heating behaviour and rheological properties of ice cream mixes. Int J Food Prop. 2005;9(4):679-689.

21. Schrosh YZ, Adam BM, phillip PS. Effect of salep as a hydrocolloid on storage stability of "Incir Uyutması" dessert. Food Hydrocoll. 2009;23(1):62-71.

22. Sophie Bourriot, Catherine Garnier, Jean-Louis Doublier. Phase separation, rheology and microstructure of micellar casein-guar gum mixtures. Food Hydrocolloids. 1999;13(1):43-39.

23. Harper F, Shoemarker M, F Naghibi, et al. Labiatae family in folk medicine in Iran: Ethnobotany to Pharmacology. J Pharma Res. 2008;4(2):63-79.

24. Buddiman OB, Watson BM. The functional, rheological and sensory characteristics of ice creams with various fat replacers. Int $J$ Dairy Technol. 2007;7:93-99.
25. Vega C, Goff HD. Phase separation in soft-serve ice mixes: Rheology and microstructure. J Int Dairy. 2008;15(3):249-254.

26. Giessen MN, Arnold RP. Kappa-carrageenan interactions in systems containing casein micelles and polysaccharide stabilizers. Food Hydrocoll. 2011;19(3):371-377.

27. Marshall RP, Rout RB, Bahram Parvar M, et al. Rheological characterization and sensory evaluation of typical soft ice cream made with selected food hydrocolloids. Food Sci Technol Int. 2013;16(1):79-88.

28. Pavia DL, Lampman GM, Kriz GS. Introduction to Spectroscopy: A Guide for Students of Organic Chemistry. 3rd ed. London: Thomson Learning Inc, Texas, USA: Fort Worth Harcourt College Publishers; 2001.

29. Jesse Greener, Bardia Abbasi, Eugenia Kumacheva. Attenuated total reflection Fourier transform infrared spectroscopy for on-chip monitoring of solute concentrations. Lab Chip. 2010;10(12):1561-1566.

30. Phillip HD, David VJ. Controlling the viscosity of ice cream mixes at pasteurization temperatures. Modern Dairy. 2000;73:12-14.

31. Thaidoum AC, Razavi BM. Heating behaviour and rheological properties of ice cream mixes. Int J Food Prop. 2005;9(4):679-689. 